Published in final edited form as:

J Cardiovasc Nurs. 2018 ; 33(6): 544-550. doi:10.1097/JCN.0000000000000498.

\title{
Cardiovascular Disease Risk Among Older Immigrants in the United States:
}

\section{A Comparison of Risk Measures}

Tina R. Sadarangani, PhD [Assistant Professor/Faculty Fellow], New York University Rory Meyers College of Nursing, New York.

Deborah Chyun, PhD [Dean and Professor], University of Connecticut School of Nursing, Storrs.

Chau Trinh-Shevrin, DrPh [Associate Professor], Department of Population Health, New York University School of Medicine, New York.

Gary Yu, PhD [Associate Research Scientist and Assistant Professor/Faculty Fellow], New York University Rory Meyers College of Nursing, New York.

Christine Kovner, PhD [Mathy Mezey Professor of Geriatric Nursing] New York University Rory Meyers College of Nursing, New York.

\section{Abstract}

Background: In the United States, 16 million immigrants are 50 years and older, but little is known about their cardiometabolic health and how to best assess their cardiovascular disease (CVD) risk. Aging immigrants may therefore not be benefitting from advances in CVD prevention.

Objective: In this study, we estimate and compare CVD risk in a nationally representative sample of aging immigrants using 3 different measures.

Methods: This was a cross-sectional analysis using National Health and Nutrition Examination Survey data. Immigrants 50 years and older with no history of CVD were eligible. The Framingham Risk Score (FRS), the American College of Cardiology/American Heart Association Pooled Cohort Risk Equation, and presence of metabolic syndrome (MetS) were used to estimate risk. Bivariate statistics were analyzed using SPSS version 23.0 Complex Survey module to account for National Health and Nutrition Examination Survey unique weighting scheme.

Results: The mean age of the sample was 61.3 years; $40 \%$ had hypertension, $17 \%$ had diabetes, $10 \%$ were smokers, and $95 \%$ did not meet the recommended physical activity guidelines. Proportions at an elevated CVD risk were as follows: American College of Cardiology/American Heart Association, $42 \%$ female and $76 \%$ male; FRS, $17.4 \%$ female and $76 \%$ male; and MetS, $22 \%$ female and $24 \%$ male.

Correspondence Tina R. Sadarangani, PhD, New York University Rory Meyers College of Nursing, 433 First Ave, Ste 645 F, New York, NY 10010, (trs233@nyu.edu). 
Conclusions: Immigrants had a lower overall risk using MetS and the American College of Cardiology/American Heart Association equation than has been found using these tools in similarly aged samples. The opposite was true for the FRS. The discrepancy between the proportion at risk and those being treated may reflect healthcare access gaps that warrant further investigation. A more holistic approach to risk measurement is needed that accounts for determinants of health that disproportionately affect immigrants, including language and socioeconomic status.

\section{Keywords}

aging; cardiovascular disease; ethnicity; immigrants; risk assessment; risk factors

Cardiovascular disease (CVD) is a problem among older adults in the United States. In the United States, adults 65 years or older represent $14.5 \%$ of the total population, yet they undergo more than $50 \%$ of cardiovascular procedures performed nationally at a cost of $\$ 121.8$ billion. ${ }^{1}$ There is strong evidence, however, that individuals who modify their lifestyles, even after the age of 50 years, can negate the effects of age-related changes to the cardiovascular system, thereby preventing costly complications. ${ }^{2,3}$

In the United States, 16 million immigrants, defined here as foreign-born aliens who intend to live abroad permanently, ${ }^{4}$ are 50 years and older. ${ }^{5}$ However, little is known about their risk of developing CVD. Although immigrants are said to be healthier than native-born individuals upon arrival into the United States, greater acculturation is associated with increases in obesity, hyperlipidemia, and cigarette smoking. ${ }^{6}$ Moreover, immigrants' health is often threatened by social factors known to heighten CVD risk, such as low socioeconomic status, limited English proficiency, and lack of access to health insurance. ${ }^{7}$

Risk reduction is the cornerstone of therapy for preventing CVD, and its complications and researchers have developed a plethora of tools intended to help clinicians calculate individuals' risk of developing CVD and its complications. ${ }^{8}$ However, many of these tools have not been validated in ethnically diverse populations, and several immigrant minority groups therefore have not benefitted from advances in treatment and prevention of CVD. ${ }^{9}$ The purpose of this study was to compare 3 measures of CVD risk in a population of middle-aged and older immigrants and estimate the proportion of those at a high risk for CVD.

\section{Outcome Measures}

Three separate tools, (1) the Framingham Risk Score (FRS), (2) the American College of Cardiology/American Heart Association (AHA) Pooled Cohort Risk Equation (PCE) for atherosclerotic cardiovascular disease, and (3) the International Diabetes Federation (IDF) definition of metabolic syndrome (MetS), were compared and used to estimate immigrants' CVD risk. The component parts of each risk measurement tool are compared with one another in Table 1.

The FRS is perhaps the most well-known measure of 10-year risk of a cardiac event. ${ }^{8,10}$ In the FRS, traditional risk factors for coronary disease (eg, age, gender) are assigned weights 
and then converted into an absolute probability of developing coronary disease. ${ }^{10}$ The $\mathrm{C}$ statistic for the FRS ranges from 0.75 to 0.8 indicating a near-excellent predictive value. ${ }^{8}$ The FRS has limitations, however. It has not been validated in diverse populations and tends to overestimate risk in minority populations. ${ }^{10,11}$ The FRS does not account for plasma glucose levels and consequently has less predictive power in individuals with type 2 diabetes mellitus (DM2). ${ }^{10}$ It also cannot predict heart or vascular diseases beyond coronary disease. ${ }^{8}$

The PCE partially addresses these shortcomings. ${ }^{12}$ It was developed using a large heterogenous multicohort sample that included African American men and women. ${ }^{12}$ The PCE predicts an individual's 10-year risk of atherosclerotic CVD (ASCVD) events, including myocardial infarction and stroke, and includes race and diabetes as risk determinants. ${ }^{12}$ It also accounts for whether an individual is being treated for hypertension and is used in the clinical setting to determine the appropriateness of initiating statin therapy in patients. ${ }^{13}$ In validation studies using racially diverse cohorts, however, the PCE demonstrated borderline/ unacceptable C-statistics, ranging from 0.56 to $0.77 .{ }^{14} \mathrm{It}$ has also been criticized for overestimating risk; in a nationally representative sample, the PCE classified $99 \%$ of people older than 70 years as needing statin therapy. ${ }^{13}$

Metabolic syndrome, defined as a cluster of risk factors, including obesity, hypertension, hyperglycemia, and dyslipidemia, has been proposed as an additional means of assessing CVD risk. ${ }^{15,16}$ It is associated with a significantly increased risk of developing DM2 and has been widely promoted as a means of identifying patients for lifestyle intervention to reduce risk factors and incident CVD, ${ }^{17,18}$ particularly in ethnically diverse populations. ${ }^{19}$ The presence of MetS has also been found to increase the risk of cerebrovascular accident (CVA) and peripheral vascular disease, which are among the complications older immigrants experience. $^{20}$

\section{Methods}

\section{Sampling}

The original data for this secondary analysis came from the National Health and Nutrition Examination Survey (NHANES) conducted by the Centers for Disease Control and Prevention. The National Health and Nutrition Examination Survey is a deidentified, publicly available data set that offers epidemiological data on the health of a nationally representative sample of community-dwelling persons in the United States. ${ }^{21}$ Participants of NHANES are identified through a complex multistage sampling strategy. ${ }^{21}$

To increase reliability, reduce variance estimates, and enable subgroup analyses, data from multiple NHANES cycles (2007-2012) were combined. For the purposes of this study, eligible participants were men and women (1) 50 years and older who were (2) born outside the United States. Combining 3 cycles of NHANES, data provided a sample of 2146 community-dwelling immigrants older than 50 years; among these, $226(10.5 \%)$ selfreported a history of cardiac disease and were excluded. 


\section{Data Collection}

The data were collected by NHANES researchers. Eligible participants provided their medical history and underwent physical examination by a trained physician, inclusive of laboratory testing at a local mobile examination center. ${ }^{21}$ National Health and Nutrition Examination Survey comprehensive laboratory and physical examination protocols are discussed elsewhere. ${ }^{22}$ Health interviews conducted in the mobile examination center were offered in multiple languages and covered a variety of topic areas. ${ }^{21}$

\section{Data Analysis}

Data were analyzed using SPSS version 23.0, a statistical analysis program, which contains a special Complex Survey module that allows users to extrapolate results to the overall US population. Subjects' data were weighted based on the unequal probability of being selected and were adjusted for nonresponse within that participant's sample category. ${ }^{23}$

The FRS, PCE, and MetS were each tabulated using standard formulas. The FRS was measured using a point-based system, an approach that is used extensively in clinical practice and endorsed by the National Cholesterol Education Project's Adult Treatment Panel III guidelines. ${ }^{11,24}$ The point-based system assigns each risk factor an integer value according to severity; the values are then summed into a cumulative gender-specific score, which corresponds to a risk proportion. ${ }^{25}$ Risk percentage scores were converted into categorical variables and categorized, consistent with clinical guidelines, as "high" ( $\geq 20 \%)$, "moderately high" $(10 \%-19 \%)$, or "moderate" $(<10 \%)$ to enhance clinical utility. ${ }^{25} \mathrm{~A}$ computerized algorithm tallied scores for individual participants in this sample.

When using the PCE, data from SPSS were exported into an AHA online spreadsheet to calculate participants' risk based on the gender-and race-specific equation. ${ }^{26}$ On the basis of results, individuals were placed in 1 of 2 categories-low/moderate risk $(<7.5)$ or high risk ( $\geq 7.5$ ) - using a computerized algorithm. A risk score of 7.5 or greater suggests moderate ASCVD risk and calls for the initiation of pharmacological therapy with statins. ${ }^{13}$

The presence or absence of MetS was determined using IDF guidelines. On the basis of IDF guidelines, participants with an increased waist circumference (which are based on ethnically specific cutoffs) - in addition to two of the following: elevated triglycerides, elevated systolic blood pressure, elevated fasting plasma glucose, or decreased high-density lipoprotein (HDL) cholesterol-were considered to have MetS. ${ }^{27}$ Because, unlike the FRS and PCE, MetS requires fasting plasma glucose measures, MetS analysis was conducted only on the fasting subset of the overall sample. Note that comparability was assessed between the fasting and nonfasting samples to ensure that there were no statistically significant differences that could potentially lead to confounding.

Preliminary descriptive statistics were used to evaluate demographic characteristics of the study population relevant to the risk tools. Measures of central tendency were calculated for age, serum laboratory values (fasting plasma glucose, HDL cholesterol, triglycerides), physical activity, and relevant components of the physical examination (systolic blood pressure, waist circumference). Continuous data were graphed using traditional scatterplots to assess linearity. The National Health and Nutrition Examination Survey complex 
sampling strategy adjusts the variability of estimates generated by the empirical study population to reflect subgroups within the national population. As a result, data dispersion was analyzed based on standard errors, as opposed to standard deviations, to enhance precision. Frequencies were used to assess categorical variables (race, gender, smoking status, etc).

\section{Results}

\section{Overall Sample Demographics}

The demographic and clinical characteristics of the final sample of immigrants 50 years and older without known coronary disease $(\mathrm{N}=1920)$ are presented in Tables 2 and 3. These tables also present stratified results for the fasting subsample used for MetS analysis.

Clinical Characteristics of the Full Sample of Older Immigrants-The mean age of the sample was 61.3 years (SE, 1.3 years). Mean values for total cholesterol (mean, 205.7 $\mathrm{mg} / \mathrm{dL} ; \mathrm{SE}, 0.01 \mathrm{mg} / \mathrm{dL}$ ), body mass index (mean, $27.8 \mathrm{~kg} / \mathrm{m}^{2} ; \mathrm{SE}, 0.06 \mathrm{~kg} / \mathrm{m}^{2}$ ), and systolic blood pressure (mean, $128.7 \mathrm{~mm} \mathrm{Hg}$; SE, $0.12 \mathrm{~mm} \mathrm{Hg}$ ) marginally exceeded healthy parameters. Mean HDL cholesterol (mean, $52.6 \mathrm{mg} / \mathrm{dL}$; SE, $0.00 \mathrm{mg} / \mathrm{dL}$ ) for the sample fell within reference limits, despite more than $95 \%$ of the study sample not meeting recommended guidelines for weekly physical activity, a known correlate of HDL cholesterol (Table 2). ${ }^{28}$

\section{Risk Factors for Cardiovascular Disease Among Immigrants $\mathbf{5 0}$ Years and} Older-Women (54\%) outnumbered men (46\%). The sample was $42.1 \%$ Hispanic and $7.0 \%$ black, and $31.3 \%$ were classified as "other," which included Asians. The remainder was white. Ten percent were current smokers. Twenty percent of the respondents described their diet as "fair" or "poor." Forty percent of the participants reported a diagnosis of hypertension, whereas $17 \%$ reported a diagnosis of DM2. The proportions of individuals on medication for hypertension (36\%) and DM2 (16\%) were slightly lower than the proportions who reported these diagnoses. Approximately, $24 \%$ of the respondents were being treated for hypercholesterolemia (Table 3).

\section{Risk Factors for Cardiovascular Disease Among Immigrants $\mathbf{5 0}$ Years and} Older-Nearly 59\% (42\% female, $76 \%$ male) were at a high ( $\geq 7.5 \%$ ) risk of ASCVD using the PCE. Smaller proportions were at an increased risk of CVD using the FRS and MetS. Using the FRS, $46 \%$ (17.4\% female, $76 \%$ male) of the sample had an elevated coronary disease risk, whereas $23 \%$ (22\% female, $24 \%$ male) had MetS (Table 4).

\section{Discussion}

This study had 2 main findings: (1) each risk measure yields a different estimate of the proportion of individuals at an elevated risk of CVD, and (2) there is a gap in the proportion of older immigrants at a high risk for CVD and those receiving pharmacologic treatment for CVD prevention. With respect to the former, the PCE estimated that $58 \%$ of older immigrants were at a high risk for ASCVD, whereas the FRS high/moderately high (48\%) and MetS (23\%) yielded lower estimates. These differences were somewhat expected given 
differences in the component parts of each outcome measure. Among the 3 outcome measures, the PCE is the most comprehensive in the risk factors it accounts for; it includes self-reported diagnosis of diabetes and treatment of hypertension in addition to clinical measures of cardiometabolic risk. This makes the PCE tool more likely than the FRS or MetS to capture those individuals who have pharmacologically controlled blood pressure and plasma glucose but have underlying diagnoses of diabetes and hypertension. Although the PCE tool also accounts for race unlike the others, this was less likely to have factored into our results given that race-specific equations only apply to African Americans. Our sample, consistent with the broader US immigrant population, was disproportionately Hispanic. ${ }^{29}$

Previous studies have found, however, that both the PCE model and FRS overestimate risk, especially in diverse samples containing Asians and Hispanics, who were not well represented in the cohorts used to derive the original tool. ${ }^{30}$ For example, in a study that compared predicted and observed CVD events using the FRS and PCE in a diverse sample from the Multi-Ethnic Study of Atherosclerosis cohort (mean age, 61 years; 53.5\% female), both the FRS and the PCE were said to have overestimated risk by as much as $154 \%$ in men and $67 \%$ in women. ${ }^{14}$

Compared with general samples of middle-aged and older adults, our findings suggest that middle-aged and older immigrants have a lower overall risk of CVD. In a similarly aged cohort of European adults, $96 \%$ of men and $66 \%$ of women were candidates for statin therapy based on the PCE. ${ }^{31}$ In our sample, the proportions were lower- $76 \%$ of men and $42 \%$ of women. However, in another study using NHANES, ${ }^{32}$ with younger (mean, 53 years old), predominately white participants, $29.9 \%$ of the participants were at an elevated risk compared with $59 \%$ overall in our study. This difference can likely be attributed to age differences between the latter sample and our own, because age is a dominant factor in 10year risk using the PCE. ${ }^{17}$

Data from a nationally representative sample of men and women aged 50 to 79 years in the United States ${ }^{33}$ showed that $82 \%$ of men and $45 \%$ of women had either moderately high or high risk of CVD using the FRS. In our study, proportions were lower using the FRS, with $76.4 \%$ of men and only $17.4 \%$ of women falling into those categories. Notably, despite different component parts, both the FRS and the PCE were in agreement on the proportion of men at a high risk but not women.

Metabolic syndrome, although arguably the least comprehensive of the 3 measures used in this study, is based entirely on objective clinical measurements and does not rely on selfreported diagnoses, which are potentially less reliable. Metabolic syndrome also does not account for the advanced age of our sample; again, age may partially explain the higher proportions of individuals at a high risk of CVD using the FRS and PCE. It is estimated that $44 \%$ of Americans 50 years and older have MetS. ${ }^{34}$ In our cohort, $23 \%$ had MetS, suggesting that our immigrant sample may have a health advantage. However, there was poor agreement between MetS and the other measures. Among those who had MetS, 42\% fell into the lowest risk category using the FRS, and 14\% were designated as low risk using the PCE. Metabolic syndrome is said to confer a 5-fold increase in the risk of type 2 
diabetes, doubles 10-year risk of CVD, and is associated with a 2- to 4-fold increase in the risk of stroke. ${ }^{35}$ Thus, inability of these other risk calculators to capture those with MetS is problematic because many of the individuals who are truly at risk based on objective clinical measures are not being treated.

Without other nationally representative samples of older immigrants to provide reference, it is unclear how accurately these tools capture older immigrants' risk and whether their risk is actually lower than general samples of adults 50 years and older. However, the failure of the PCE tool, in particular, to capture those with MetS may reflect the fact that many immigrants, especially women, are unaware as to whether they have diabetes or hypertension, which are components of this calculator. Immigrant populations have been found to demonstrate low levels of CVD risk factor awareness and are less likely to receive treatment. ${ }^{36}$

There were minor differences between the percentage of those with diagnoses of hypertension and DM and the percentage receiving treatment of those disorders. However, the discrepancy was greater with respect to blood cholesterol levels. Although $42 \%$ of the sample self-reported a diagnosis of hypercholesterolemia, only $24 \%$ were receiving medication. Or, using the FRS and PCE categorizations, which are indications for cholesterol-lowering therapy, less than half of individuals who qualified for statin therapy actually reported getting it. This may be a reflection of health access barriers. It may also be a consequence of racial bias that has historically contributed to disparities in the quality and intensity of care for minorities. ${ }^{37,38}$ This area warrants further analysis among aging immigrants.

This study has several limitations, beyond the crosssectional design that prohibits causal inference. The data are limited by reliance on participant self-report without corroboration from a formal medical record. This has implications for prevalence estimates. For example, the proportion of persons with hypertension and DM is based on self-reported diagnoses and awareness of these conditions. This is known to be problematic in immigrant populations, where health advantages are illusory and partially attributable to diseases that go undiagnosed in the sending country. ${ }^{39}$ Thus, rates of hypertension and DM may be higher than reported. The most significant limitation was the inability to capture meaningful clinical differences among racial/ethnic subgroups using these particular cycles of NHANES data. This is especially important in light of the fact that Hispanics and Asians represent two of the largest immigrant groups in the United States, and there is significant heterogeneity in clinical risk between them. ${ }^{40}$ Moreover, country of origin was not assessed in 2 of 3 NHANES cycles. Whereas Hispanics were oversampled in all 3 data cycles, Asians were only oversampled in 1 cycle of data, and proportions were too small to support subgroup analysis. Aggregating Asians and Hispanics may have inadvertently biased the findings.

\section{Conclusion}

The authors estimated CVD risk factors in a diverse population using 3 different measures and uniquely provided population estimates of the proportion of middle-aged and older immigrants at risk of heart disease in the United States. This study suggests that, in 
immigrants, mainstream measures of CVD risk are not reliable when used in isolation. None of the 3 outcome measures used in this study fully captures individuals' risk of CVD. Rather, clinicians need to analyze the estimates and indications put forth by the PCE, FRS, and MetS in a holistic context. Not only does CVD risk need to be explored using objective clinical measures, including the increasingly used coronary artery calcification scan, but also within the context of patients' family and social history. Other factors that may heighten immigrants' health risks and reduce the likelihood that they are receiving appropriate therapy, including low socioeconomic status, limited English proficiency, and discrimination by providers, must also be included as part of immigrants' broader CVD risk assessment.

\section{Acknowledgments}

This study was funded by the National Hartford Centers for Gerontological Nursing Excellence. Dr Sadarangani's research was supported by the National Hartford Centers of Gerontological Nursing Excellence Patricia G. Archbold Scholar Award Program. The authors have no conflicts of interest to disclose.

\section{REFERENCES}

1. Mozaffarian D, Benjamin EJ, Go AS, et al. Heart disease and stroke statistics-2015 update: a report from the American Heart Association. Circulation. 2015;131(4):e29-e322. [PubMed: 25520374]

2. Larsen P. A review of cardiovascular changes in the older adult. Gerontology Update. https:// www.rehabnurse.org/pdf/GeriatricsCV.pdf. Accessed August 1, 2017.

3. Berry JD, Dyer A, Cai X, et al. Lifetime risks of cardiovascular disease. N Engl J Med. 2012;366(4): 321-329. [PubMed: 22276822]

4. Department of Homeland Security. Immigration data \& statistics. Definition of terms. https:// www.dhs.gov/immigration-statistics/data-standards-and-definitions/definitionterms\#permanent_resident_alien. Accessed January 1, 2018.

5. US Census Bureau. Characteristics of the foreign-born population by nativity and US citizenship status. Current Population Survey. https://www.census.gov/data/tables/2013/demo/foreign-born/ cps-2013.html. Accessed August 1, 2017.

6. Kennedy S, Kidd MP, McDonald JT, Biddle N. The healthy immigrant effect: patterns and evidence from four countries. JIMI. 2015;16(2):317-332.

7. Edberg M, Cleary S, Vyas A. A trajectory model for understanding and assessing health disparities in immigrant/refugee communities. J Immigr Minor Health. 2011;13:576-584. [PubMed: 20306225]

8. Lloyd-Jones DM. Cardiovascular risk prediction: basic concepts, current status, and future directions. Circulation. 2010;121(15):1768-1777. [PubMed: 20404268]

9. Havranek EP, Mujahid MS, Barr DA, et al. Social determinants of risk and outcomes for cardiovascular disease: a scientific statement from the American Heart Association. Circulation. 2015;132(9):873-898. [PubMed: 26240271]

10. Hemann BA, Bimson WF, Taylor AJ. The Framingham Risk Score: an appraisal of its benefits and limitations. Am Heart Hosp J. 2007;5(2):91-96. [PubMed: 17478974]

11. The National Cholesterol Education Program. Executive summary of the third report of the National Cholesterol Education Program (NCEP) Expert Panel on Detection. Evaluation and treatment of high blood cholesterol in adults. JAMA. 2001;285(19):2486-2497. [PubMed: 11368702]

12. Preiss D, Kristensen SL. The new pooled cohort equations risk cohort. Can J Cardiol. 2015;31(5): 613-619. [PubMed: 25843167]

13. KarmaliKN GoffDC, NingH Lloyd-JonesDM. A systematic examination of the 2013 ACC/AHA Pooled Cohort Risk Assessment Tool for Atherosclerotic Cardiovascular Disease. J Am Coll Cardiol. 2014;64(10):959-968. [PubMed: 25190228] 
14. DeFilippis AP, Young R, Carrubba CJ, et al. An analysis of calibration and discrimination among multiple cardiovascular risk scores in a modern multiethnic cohort. Ann Intern Med. 2015;162(4): 266-275. [PubMed: 25686167]

15. Alexander CM, Landsman PB, Teutsch SM, Haffner SM. Third National Health and Nutrition Examination Survey (NHANES III); National Cholesterol Education Program (NCEP). NCEPdefined metabolic syndrome, diabetes, and prevalence of coronary heart disease among NHANES III participants age 50 years and older. Diabetes. 2003;52(5): 1210-1214. [PubMed: 12716754]

16. Kurth T, Logroscino G. The metabolic syndrome: more than the sum of its components? Stroke. 2008;39:1068-1069. [PubMed: 18323482]

17. Grundy SM. Pre-diabetes, metabolic syndrome, and cardiovascular risk. J Am Coll Cardiol. 2012;59(7):635-643. [PubMed: 22322078]

18. Meigs J. Metabolic syndrome: in search of a clinical role. Diabetes Care. 2004;27(11):2761-2763. [PubMed: 15505020]

19. Khanna R, Kapoor A, Kumar S, et al. Metabolic syndrome \& Framingham Risk Score: observations from a coronary angiographic study in Indian patients. Indian J Med Res. 2013;137:295-301. [PubMed: 23563372]

20. Argeseanu-Cunningham S, Ruben JD, Venkat-Narayan KM. Health of foreign-born people in the United States: a review. Health Place. 2008;14:623-635. [PubMed: 18242116]

21. Centers for Disease Control and Prevention. National Health and Nutrition Examination Survey. National Center for Health Statistics https://www.cdc.gov/nchs/nhanes/index.htm. Accessed August 1, 2017.

22. Centers for Disease Control and Prevention. National Health and Nutrition Examination Survey (NHANES) laboratory procedures manual. National Center for Health Statistics http:// www.cdc.gov/nchs/data/nhanes/nhanes_09_10/lab.pdf. Accessed August 1, 2017.

23. Curtin LR, Mohadjer LK, Dohrmann SM, et al. National Health and Nutrition Examination Survey: sample design, 2007-2010. Vital Health Stat 2 2013;(160):1-23.

24. Gordon WJ, Polansky JM, Boscardin WJ, Fung KZ, Steinman MA. Coronary risk assessment by point-based vs. equation-based Framingham models: significant implications for clinical care. J Gen Intern Med. 2010;25(11):1145-1151. [PubMed: 20824362]

25. Wilson PW, D’Agostino RB, Levy D, Belanger AM, Silbershatz H, Kannel WB. Prediction of coronary heart disease using risk factor categories. Circulation. 1998;97:1837-1847. [PubMed: 9603539]

26. Goff DC,Lloyd-Jones DM,Bennett G,et al. 2013 ACC/AHA Guideline on the Assessment of Cardiovascular Risk. Circulation. 2014;129(suppl 2):S49-S73. [PubMed: 24222018]

27. Ford ES. Prevalence of the metabolic syndrome defined by the International Diabetes Federation among adults in the U.S. Diabetes Care. 2005;28(11):2745-2749. [PubMed: 16249550]

28. Braun LT, Rosenson RS. Effects of exercise on lipoproteins and hemostatic factors. UptoDate. http://www.uptodate.com/contents/effects-of-exercise-on-lipoproteins-and-hemostatic-factors. Accessed August 1, 2017.

29. Lopez G, Bialik K. Key findings about US immigrants. http://www.pewresearch.org/fact-tank/ 2017/05/03/key-findings-about-u-s-immigrants/. Accessed October 25, 2017.

30. Smith SC, Grundy SM. 2013 ACC/AHA guideline recommends fixed-dose strategies instead of targeted goals to lower blood cholesterol. J Am Coll Cardiol. 2014;64(6):601-612. [PubMed: 25104531]

31. Kavousi M, Leening MJ, Nanchen D, et al. Comparison of application of the ACC/AHA Guidelines, Adult Treatment Panel II Guidelines, and European Society of Cardiology Guidelines for cardiovascular disease prevention in a European cohort. JAMA. 2014;311(14):1416-1423. [PubMed: 24681960]

32. Nooe A, Edwards MK, Addoh O, Loprinzi P. Convergent validity of the ACC/AHA pooled cohort equations in associating with health-related quality of life among adults in the United States. Health Promot Perspect. 2017;7(1):42-46. [PubMed: 28058241]

33. Ford ES, Giles WH, Mokdad AH. The distribution of 10-year risk for coronary heart disease among US adults: findings from the National Health and Nutrition Examination Survey III. J Am Coll Cardiol. 2004;43(10):1791-1796. [PubMed: 15145101] 
34. Centers for Disease Control and Prevention. Metabolic syndrome prevalence by race/ethnicity and sex in the United States, National Health and Nutrition Examination Survey, 1988-2012.

Preventing Chronic Disease. https://www.cdc.gov/pcd/issues/2017/16_0287a.htm. Accessed August 1, 2017.

35. Kaur J. A comprehensive review on metabolic syndrome. Cardiol Res Pract. 2014:1-21. doi: $10.1155 / 2014 / 943162$.

36. Langellier BA, Garza JR, Glik D, et al. Immigration disparities in cardiovascular disease risk factor awareness. J Immigr Minor Health. 2012;14(6):918-925. [PubMed: 22210443]

37. Derose KP, Escarce JJ, Lurie N. Immigrants and health care: sources of vulnerability. Health Aff. 2007;26(5): 1258-1268.

38. Williams DR, Wyatt R. Racial bias in healthcare and health: challenges and opportunities. JAMA. 2015;314(6): 555-556. [PubMed: 26262792]

39. Barcellos SH, Goldman DP, Smith JP. Undiagnosed disease, especially diabetes, casts doubt on some of reported health 'advantage' of recent Mexican immigrants. Health Aff. 2012;31(12): 2727-2737.

40. Guerrero AD, Chung PJ. Racial and ethnic disparities in dietary intake among California children. J Acad Nutr Diet. 2015;116(3):439-448. [PubMed: 26433453] 


\section{What's New and Important}

- As many as $59 \%$ of aging immigrants are at a heightened risk of CVD, but a much smaller proportion is receiving appropriate treatment.

- Widely used measures of cardiovascular risk, including the new American College of Cardiology/AHA PCE, may not fully capture aging immigrants' risk of CVD because they (1) rely heavily on self-report in a population that has low levels of awareness of CVD risk factors and (2) do not address social disadvantages that may elevate risk. 


\section{TABLE 1}

\section{Comparison of Risk Measurement Tool Components}

\begin{tabular}{lccc} 
& FRS & MetS & PCE \\
\hline Includes age & $\mathrm{X}^{a}$ & & $\mathrm{X}^{a}$ \\
Includes gender & $\mathrm{X}^{a}$ & & $\mathrm{X}^{a}$ \\
Includes current smoking & $\mathrm{X}^{a}$ & & $\mathrm{X}^{a}$ \\
Includes systolic blood pressure & $\mathrm{X}^{b}$ & $\mathrm{X}^{b, c}$ & $\mathrm{X}^{b}$ \\
Includes plasma glucose & & $\mathrm{X}^{a}{ }^{a}$ & \\
Includes HDL cholesterol & $\mathrm{X}^{d}$ & $\mathrm{X}^{a}{ }^{c}{ }^{c}{ }^{d}$ & $\mathrm{X}^{d}$ \\
Includes total cholesterol & $\mathrm{X}^{d}$ & & $\mathrm{X}^{d}$ \\
Includes triglycerides & & $\mathrm{X}^{d}$ & \\
Includes waist circumference & & $\mathrm{X}^{b}$ & \\
Includes race & & & $\mathrm{X}^{a}$ \\
History of diabetes & & & $\mathrm{X}^{a c}{ }^{a}$ \\
Includes treatment of hypertension & & & $\mathrm{X}^{a c}{ }^{c}$ \\
\hline
\end{tabular}

Abbreviations: FRS, Framingham Risk Score; HDL, high-density lipoprotein; MetS, metabolic syndrome; PCE, Pooled Cohort Risk Equation.

a Based on self-report.

$b_{\text {Based on clinical examination. }}$

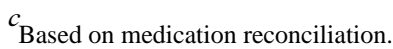

$d_{\text {Based on serum laboratory values. }}$ 
TABLE 2

Clinical Characteristics of Immigrants 50 Years and Older (Adjusted)

\begin{tabular}{|c|c|c|c|c|}
\hline \multirow[b]{2}{*}{ Category } & \multicolumn{2}{|c|}{$\begin{array}{c}\text { Total Sample } \\
(\mathbf{N}=\mathbf{1 9 2 0})\end{array}$} & \multicolumn{2}{|c|}{$\begin{array}{c}\text { Fasting } \\
\text { Subsample } \\
(\mathbf{n}=977)\end{array}$} \\
\hline & Mean & SE & Mean & SE \\
\hline Age, y & 61.3 & 0.12 & $61.6^{a}$ & 0.11 \\
\hline Total cholesterol, mg/dL & 205.7 & 0.01 & 204.9 & 0.02 \\
\hline Body mass index, $\mathrm{kg} / \mathrm{m}^{2}$ & 27.8 & 0.06 & 27.9 & 0.09 \\
\hline Systolic BP, mm Hg & 128.7 & 0.12 & $128.0^{a}$ & 0.33 \\
\hline Diastolic BP, mm Hg & 70.8 & 0.18 & $66.9^{a}$ & 0.33 \\
\hline HDL-C, mg/dL & 52.6 & 0.00 & 53.8 & 0.00 \\
\hline Fasting plasma glucose, $\mathrm{mg} / \mathrm{dL}$ & N/A & N/A & 111.6 & 0.24 \\
\hline Waist circumference, $\mathrm{cm}$ & 94.7 & 0.13 & 95.5 & 0.20 \\
\hline Triglycerides, mg/dL & N/A & N/A & 139.1 & 0.99 \\
\hline
\end{tabular}

Abbreviations: BP, blood pressure; HDL-C, high-density lipoprotein cholesterol; N/A, not applicable (fasting participants only). ${ }^{a} P<.001$. 
TABLE 3

Risk Factors for Cardivascular Disease Among Immigrants 50 Years and Older (Adjusted)

\begin{tabular}{lcc} 
Category & $\begin{array}{c}\text { Total Sample } \\
(\mathbf{N}=\mathbf{1 9 2 0}), \%\end{array}$ & $\begin{array}{c}\text { Fasting } \\
\text { Subsample } \\
(\mathbf{n}=\mathbf{9 7 7}), \%\end{array}$ \\
\hline Gender (female) & 54 & 53 \\
Hispanic & 42.1 & $43.2^{a}$ \\
White & 19.6 & 20.7 \\
Black & 7.0 & 7.1 \\
Other (including Asian American, multiracial) & 31.3 & 29.1 \\
Residing in the United States for $>10$ y & 87 & 87 \\
Current smokers & 10 & 10 \\
Self-reported diet is fair or poor & 20 & 20 \\
Do not meet recommended PA guidelines $\dagger$ & 95 & 93 \\
Hypertension (self-reported) & 40 & 40 \\
Diabetes mellitus (self-reported) & 17 & 20 \\
Takes medication for hypertension & 36 & 37 \\
Takes medication for hypercholesterolemia & 24 & 24 \\
Takes oral medication for diabetes mellitus & 16 & 16 \\
Takes insulin for diabetes mellitus & 3 & 3 \\
\hline
\end{tabular}

Abbreviation: PA, physical activity.

${ }_{P}^{a}<.001$. 
TABLE 4

Overall Risk for Cardiovasular Disease by Measure Among Male and Female Immigrants 50 Years and Older (Adjusted)

\begin{tabular}{|c|c|c|}
\hline $\begin{array}{l}\text { Cardiovascular Risk } \\
\text { Measure }\end{array}$ & $\begin{array}{l}\text { Total Sample } \\
(\mathrm{N}=1920), \%\end{array}$ & $\begin{array}{c}\text { Fasting } \\
\text { Subsample } \\
(\mathrm{n}=977), \%\end{array}$ \\
\hline \multicolumn{3}{|l|}{$\mathrm{PCE}>7.5 \%$} \\
\hline Overall & 59 & 59 \\
\hline Male & 76 & 78 \\
\hline Female & 42 & 40.9 \\
\hline \multicolumn{3}{|c|}{ Framingham Risk Score, moderately high (10\%-19\%) } \\
\hline Overall & 31 & 30 \\
\hline Male & 47.4 & 45 \\
\hline Female & 13.6 & 16.4 \\
\hline \multicolumn{3}{|c|}{ Framingham Risk Score, high $(>20 \%)$} \\
\hline Overall & 16 & 17 \\
\hline Male & 29 & 32 \\
\hline Female & 3.8 & 2.7 \\
\hline \multicolumn{3}{|l|}{ Metabolic syndrome } \\
\hline Overall & N/A & 23 \\
\hline Male & N/A & 24 \\
\hline Female & N/A & 22 \\
\hline
\end{tabular}

Abbreviations: N/A, not applicable (fasting participants only); PCE, Pooled Cohort Risk Equation. 